\title{
Membrane-associated proteins encoded by the nisin gene cluster may function as a receptor for the lantibiotic carnocin U149
}

\author{
Geesje Stoffels, ${ }^{1,2}$ Ágústa Guðmundsdóttir ${ }^{1}$ and Tjakko Abee ${ }^{2}$ \\ Author for correspondence: Tjakko Abee. Tel: +318370 84981. Fax: +318370 84893. e-mail: \\ Tjakko.Abee@LMC.LMT.WAU.NL
}

1 Science Institute, University of Iceland, Dunhaga 3, 107 Reykjavík, Iceland

2 Department of Food Science, Wageningen Agricultural University, Bomenweg 2, POB 8129, 6700 EV Wageningen, The Netherlands

\begin{abstract}
Carnocin U149, a lantibiotic produced by Carnobacterium piscicola shows bactericidal activity against many lactic acid bacteria. This paper describes the results of a study on the mode of action of carnocin U149. It has previously been observed that nisin-producing Lactococcus lactis subsp. lactis strains are at least 10-fold more sensitive to carnocin U149 relative to other lactic acid bacteria. Addition of carnocin U149 to cells of L. lactis subsp. lactis NZ9700 resulted in a dissipation of the membrane potential and a rapid hydrolysis of internal ATP. These results suggest that carnocin U149 may act at the cytoplasmic membrane. The correlation between production of and/or immunity to nisin and sensitivity to carnocin of $L$. lactis subsp. lactis strains was further investigated. Several transformed $L$. lactis subsp. lactis strains carrying a fragment of the nisin gene cluster were tested for their sensitivity to carnocin U149 and their immunity to nisin. The results suggest that NisP, which is one of the membrane-associated proteins involved in the production of nisin acts as receptor for carnocin U149. This may facilitate the binding and/or insertion of carnocin UI49 into the cytoplasmic membrane thus increasing its bactericidal activity. Lantibiotic-producing and non-producing mutants of Staphylococcus epidermidis and Lactobacillus sake did not show a difference in sensitivity to carnocin U149. The proposed receptor-mediated action of carnocin U149 at the cytoplasmic membrane therefore seems to be specific for the nisin-producing strains.
\end{abstract}

Keywords: Carnobacterium piscicola, lactic acid bacteria, lantibiotic, carnocin UI49, receptor

\section{INTRODUCTION}

The ability of lactic acid bacteria (LAB) to inhibit the growth of other bacteria is well known. One factor responsible for this inhibition is the production of antimicrobial peptides, the so-called bacteriocins. Bacteriocins are produced by all genera of LAB and many bacteriocins have been isolated and characterized. Bacteriocins produced by LAB have recently been reviewed (Hoover \& Steenson, 1993, Klaenhammer, 1993).

A small number of bacteriocins have been the subject of extensive research due to their potential use as antimicrobial agents in food. In this context, one class of bacteriocins, the lantibiotics, is of special interest, because

Abbreviations: $\mathrm{LAB}$, lactic acid bacteria; TPP+, tetraphenylphosphonium cation. these exert a broader spectrum of antimicrobial activity than many other bacteriocins. Lantibiotics consist of a polypeptide chain that has been post-translationally modified. These bacteriocins contain the dehydrated amino acids dehydroalanine (Dha) and dehydrobutyrine (Dhb) as well as lanthionine and $\beta$-methyl-lanthionine which are formed after S-ring formation of cysteine with Dha and Dhb respectively (Jung, 1991). These unusual amino acids may play an important role in the bactericidal activity of these peptides (Kuipers et al., 1993).

Nisin was the first bacteriocin permitted as a food additive and is now used as such in at least 46 countries. It has been shown to inhibit Clostridium species in processed cheeses, dairy products and canned foods (Anon., 1989; DelvesBroughton, 1990). Nisin is active and stable under acidic conditions. It is therefore not the preservative of choice for meat and fish because these are not acid environments. 
Table 1. Characteristics of the indicator strains

\begin{tabular}{|c|c|c|c|}
\hline Indicator strain & $\begin{array}{c}\text { Relevant } \\
\text { characteristics }\end{array}$ & $\begin{array}{c}\text { Extracellular } \\
\text { product }\end{array}$ & Reference \\
\hline \multicolumn{4}{|l|}{ L. lactis subsp. lactis } \\
\hline NZ9700 & $\operatorname{Tn} 5276$ & Nisin A & Kuipers et al. (1993) \\
\hline R520 & Mutated $\operatorname{Tn} 5276$ & - & Rauch et al. (1991) \\
\hline MG1614(pNZ9111) & nis $A B T C I R$ & Precursor nisin A & Kuipers et al. (1993) \\
\hline NZ9800 & $\operatorname{Tn} 5276 \Delta$ nis A & - & Kuipers et al. (1993) \\
\hline MG1614 & - & - & Gasson (1983) \\
\hline Lb. sake $\mathbf{L} 45$ & & & Mortvedt et al. (1991) \\
\hline LMG2340 & pCIM1 & Lactocin S & \\
\hline LMG2346 & - & - & \\
\hline S. epidermidis & & & Reis \& Sahl (1991) \\
\hline 5 & pED503 & Pep5 & \\
\hline $5 \mathrm{Pep}^{-}$ & Lacks pED503 & - & \\
\hline $5 \mathrm{Pep}^{-}(\mathrm{pMR} 2)$ & $\begin{array}{l}\text { Contains fragment of } \\
\text { pED503 }\end{array}$ & prePep5 & \\
\hline
\end{tabular}

Therefore, there is a need for novel lantibiotics with unique properties. Three lantibiotics have recently been isolated: lactocin S (Mørtvedt et al., 1991), lacticin 481 (Piard et al., 1992) and carnocin UI49 (Stoffels et al., 1992a). In order to exploit the potential of these lantibiotics, research is required on their structure, mode of action and genetics.

This paper presents results on the mode of action of carnocin UI49, a polypeptide containing a large number of hydrophobic amino acid residues (Stoffels et al., 1992a) as well as a cationic C-terminal tail (unpublished results). The amphiphilic character of this bacteriocin suggests that the primary target may be the cytoplasmic membrane. Therefore, the effect of carnocin UI49 on the permeability of the cytoplasmic membrane of sensitive cells was studied. In these experiments, the indicator organism was a nisin-producing Lactococcus lactis subsp. lactis strain. It had previously been observed that these strains are at least 10 -fold more sensitive to carnocin UI49 than other LAB. Thus, the activity of carnocin UI49 was tested against various transformed $L$. lactis subsp. lactis strains carrying a fragment of the nisin gene cluster. A mechanism of action for carnocin UI49 is proposed in this paper based on the genetic characteristics and the phenotype of these strains and their sensitivity to carnocin UI49.

\section{METHODS}

Bacterial cultures and media. Carnobacterium piscicola UI49, the carnocin UI49 producer strain was isolated from fish (Stoffels $e t$ al., 1992a). Lactococcus lactis subsp. lactis SIK-83, the nisin Z producer strain (De Vos et al., 1993) was isolated from lactic acid fermented carrots (Andersson, 1986) and kindly provided by Rolf Andersson, Swedish Food Institute, Gothenburg, Sweden.

L. lactis subsp. lactis strains were kindly supplied by Jeroen Hugenholtz and Oscar Kuipers, Department of Microbiology, Netherlands Institute for Dairy Research (NIZO), Ede, The Netherlands. The lactocin-S-producing Lactobacillus sake strain and its mutant were obtained from Ingolf Nes, Laboratory of Microbial Gene Technology, Ås, Norway. The Pep5-producing Stapbylococcus epidermidis strain and its mutants were obtained from Hans-Georg Sahl, Institute for Medical Microbiology and Immunology, University of Bonn, Germany. The characteristics of the strains are presented in Table 1.

Cultures were maintained as frozen stocks in $20 \%(\mathrm{v} / \mathrm{v})$ glycerol at $-75^{\circ} \mathrm{C}$. C. piscicola UI49 and the lactococci were propagated in BHI supplemented with $0.5 \%(\mathrm{w} / \mathrm{v})$ glucose (GBHI). $L$. lactis subsp. lactis MG1614(pNZ9111) was grown in GBHI in the presence of $10 \mu \mathrm{g}$ erythromycin $\mathrm{l}^{-1}$ (Sigma). Lb. sake strains were propagated in MRS. All media were obtained from Difco.

Purification of carnocin U149 and nisin Z. Carnocin UI49 from C. piscicola UI49 and nisin Z from L. lactis subsp. lactis SIK-83 were purified from $1 \mathrm{l}$ cultures in GM17 as described previously (Stoffels et al., 1993). Protein concentrations were estimated by comparison with a Coomassie Blue binding assay (Zaman \& Verwilghen, 1979) using a mixture of human albumin and globulin (5:3) (Sigma) as a standard.

Bacteriocin assay. The sensitivity of the indicator strains to carnocin UI49 and nisin Z was measured with the microtitre plate assay system as described previously (Mørtvedt et al., 1991; Stoffels et al., 1992a, b). The nisin-producing strain $L$. lactis subsp. lactis NZ9700 was sensitive to minimal $0.09 \mathrm{mg}$ carnocin UI49 $\mathrm{l}^{-1}$ and immune to maximal $3.3 \mathrm{mg}$ nisin $\mathrm{Z}^{-1}$. These values were used as references for the other $L$. lactis subsp. lactis strains.

The well diffusion assay as described by Stoffels et al. (1992b) was used to measure the activity of carnocin UI49 at $12{ }^{\circ} \mathrm{C}$ and $30^{\circ} \mathrm{C}$. Purified carnocin UI49 $\left(0.19 \mathrm{mg} \mathrm{m}^{-1}\right)$ was added to $5 \mathrm{~mm}$ diameter wells in BHI agar plates. Carnocin UI49 was allowed to diffuse into the agar at room temperature $(3-4 \mathrm{~h}) . L$. lactis subsp. lactis NZ9700, the indicator strain, was preincubated twice at $30^{\circ} \mathrm{C}$ or $12{ }^{\circ} \mathrm{C}$. BHI soft agar $(0.7 \%$, w/v, agar $)$ was seeded with $L$. lactis subsp. lactis NZ9700 and poured over the surface of the agar well plates. After incubation of $24 \mathrm{~h}$ at $30^{\circ} \mathrm{C}$ or $48 \mathrm{~h}$ at $12^{\circ} \mathrm{C}$ the diameters of the inhibition zones were measured.

Measurement of the membrane potential. Cells of the indicator strain L. lactis subsp. lactis NZ9700, grown overnight at $30^{\circ} \mathrm{C}$ in $\mathrm{GBHI}$, were diluted 10 -fold in fresh medium and 
grown to an optical density at $620 \mathrm{~nm}$ of 0.9 . Cells were harvested by centrifugation, washed once in $50 \mathrm{mM}$ potassium phosphate buffer, $\mathrm{pH} 6.0$, and resuspended in the same buffer to an optical density at $620 \mathrm{~nm}$ of approximately 20 . Cells were stored on ice and used within $3 \mathrm{~h}$. The experiment was performed at $30^{\circ} \mathrm{C}$. The incubation mixture was composed of $0 \cdot 1 \mathrm{ml}$ of cell suspension, $1.0 \mathrm{ml} 50 \mathrm{mM}$ potassium phosphate buffer, pH 6.0, containing glucose $(0 \cdot 18 \%)$ and the lipophilic cation tetraphenylphosphonium $\left(\mathrm{TPP}^{+} ; 3.6 \mu \mathrm{M}\right)$. By addition of the $\mathrm{K}^{+} / \mathrm{H}^{+}$exchanger nigericin $(0.36 \mu \mathrm{M}$, final concentration), the $\mathrm{pH}$ gradient (alkaline inside) was dissipated such that the protonmotive force was composed of the membrane potential only (Driessen et al., 1987). The change in membrane potential was monitored by the distribution of $\mathrm{TPP}^{+}$using a $\mathrm{TPP}^{+}$selective electrode (Shinbo et al., 1978).

Measurement of hydrolysis and/or efflux of ATP. The ATP content of cell suspensions was determined by the luciferaseluciferin procedure with an ATP bioluminescence assay kit (Lumac). A cell suspension of L. lactis subsp. lactis NZ9700 was prepared as described above. The experiment was performed at $30^{\circ} \mathrm{C}$. The incubation mixture was composed of $0.2 \mathrm{ml}$ of cell suspension and $2.8 \mathrm{ml} 50 \mathrm{mM}$ potassium phosphate buffer, $\mathrm{pH} 6.0$, containing $0.5 \%$ glucose. Aliquots from the mixture with or without carnocin UI49 $\left(6.3 \mathrm{mg} \mathrm{l}^{-1}\right)$ were taken at regular time intervals for determination of ATP. For determination of the total (internal and external) amount of ATP, $20 \mu \mathrm{l}$ of the cell suspension was rapidly lysed by the addition of $80 \mu \mathrm{l}$ dimethylsulfoxide. The suspensions were diluted in $5.0 \mathrm{ml}$ sterile water. An aliquot of $0.2 \mathrm{ml}$ of the suspension or a dilution thereof was mixed with the luciferin-luciferase reagent and ATP determined with the Lumac luminometer. To determine the extracellular ATP concentration, $20 \mu \mathrm{l}$ was diluted with $5.0 \mathrm{ml} 50 \mathrm{mM}$ potassium phosphate buffer, $\mathrm{pH} 6.0$, and $0.1 \mathrm{ml}$ was analysed as described above. The assays were calibrated using ATP solutions of known concentrations.

\section{RESULTS AND DISCUSSION}

\section{Effect of carnocin UI49 on the permeability of the cytoplasmic membrane of the nisin-producing $L$. lactis subsp. lactis NZ9700}

The wide spectrum of activity as well as the amphiphilic character of carnocin UI49 suggest that it acts on the cytoplasmic membrane in a similar fashion to nisin. Nisin acts at the cytoplasmic membrane by forming pores in the membrane. This pore formation is described in the socalled barrel-stave mechanism. The water soluble monomeric bacteriocin molecules bind and subsequently insert into the membrane. Aggregation of the molecules like barrel staves results in pore formation. The hydrophobic side of the protein is exposed to the membrane acyl chains and the hydrophilic sides line the pore. As a result, stable transmembrane pores are formed that allow the passive flux of ions and small molecules across the bilayer thereby killing the cell (Ruhr \& Sahl, 1985; A bee et al., 1991; Benz et al., 1991; Ojcius \& Young, 1991; Garcera et al., 1993; Venema et al., 1993). The broad spectrum of activity of nisin suggests that it does not require a specific integral membrane receptor for insertion (Ray, 1992). This was confirmed by the observation that nisin also acts on liposomes (Benz et al., 1991; Gao et al., 1991). The nisin activity is influenced by the phospholipid composition of the liposomal membrane, which offers an explanation for the differences in sensitivity of bacterial species to nisin (Gao et al., 1991; Garcera et al., 1993).

The membrane potential of sensitive nisin-producing lactococcal cells was measured after addition of carnocin UI49 to test the activity of carnocin UI49 on the cytoplasmic membrane. Energized cells of $L$. lactis subsp. lactis strain NZ9700 retained a membrane potential of $-124 \mathrm{mV}$ (negative inside) at $\mathrm{pH} 6$ in the presence of nigericin $(0.36 \mu \mathrm{M})$ which could be dissipated by valinomycin $(0.72 \mu \mathrm{M})$ (Fig. 1). In a second experiment, addition of carnocin UI49 $\left(8.5 \mathrm{mg} \mathrm{l}^{-1}\right.$, final concentration) also resulted in complete depolarization of the cytoplasmic membrane (Fig. 1). No further dissipation of the membrane potential was observed when valinomycin $(0.72 \mu \mathrm{M})$ was added to the cells after treatment with carnocin UI49 (data not shown).

The increase in membrane permeability caused by carnocin UI49 was further studied by measuring the hydrolysis and/or efflux of ATP after addition of carnocin UI49. When the starved lactococcal cells were energized with glucose, the ATP concentration increased significantly (Fig. 2). Carnocin UI49 (6.3 $\mathrm{mg} \mathrm{l}^{-1}$, final concentration) was added to the cell suspension when $50 \%$ or $100 \%$ of the total ATP had been produced by the cells (Fig. 2a and $\mathrm{b}$, respectively). ATP production ceased immediately after addition of carnocin UI49 to the cells (Fig. 2a). This suggests that carnocin has an immediate effect on the cells. ATP hydrolysis was observed after a short lagtime (Fig. $2 \mathrm{a}, \mathrm{b})$. Interestingly, the decrease in the $\mathrm{pH}$ of the cell suspension which normally drops from 6 to approximately $4 \cdot 2$ stopped immediately after addition of carnocin UI49 (data not shown). This is a further indication of inhibition of the sugar metabolism of the cells.

Both the membrane potential dissipation and ATP hydrolysis by cells of $L$. lactis subsp. lactis NZ9700 indicate that the cytoplasmic membrane is the primary target for carnocin UI49. The bacteriocin may form pores according to the barrel-stave mechanism of action as described for nisin. In this study, no efflux of ATP was observed (data not shown) which suggests that the pores are very small. However, if carnocin acts in the same fashion as nisin, the pores are formed upon oligomerization of carnocin UI49 molecules in the membrane. A higher bacteriocin concentration may therefore result in larger pore sizes resulting in the efflux of larger compounds such as ATP (Sahl et al., 1987; Benz et al., 1991).

The ATP hydrolysis caused by carnocin UI49 may be induced upon dissipation of the protonmotive force, i.e. increased ATP consumption due to higher activity of the $\mathrm{H}^{+}$-ATPase. The ATP hydrolysis may also be due to the efflux of phosphate and subsequent shift in the ATP hydrolysis equilibrium as was recently described for the action of pore-forming colicins and phage T5 against Escherichia coli (Guihard et al., 1993).

\section{Effect of temperature on the activity of carnocin U149}

The effect of temperature on the activity of carnocin UI49 has been studied previously (Stoffels et al., 1992b). In those studies, the sensitive strain $L$. lactis subsp. lactis 


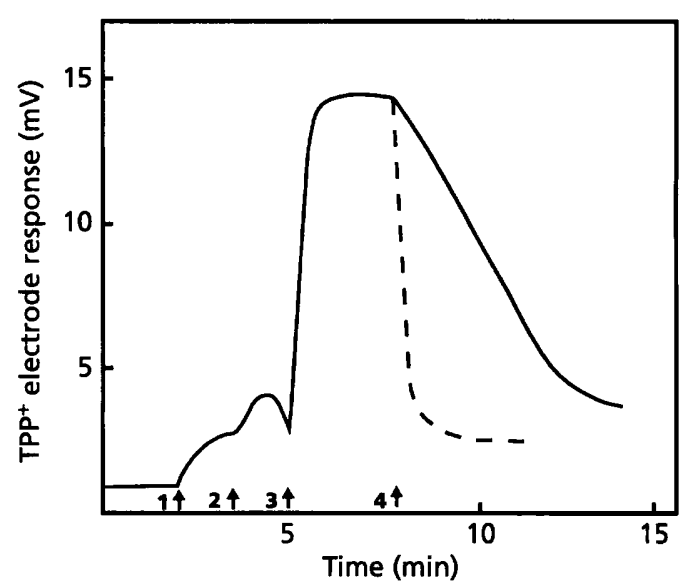

Fig. 1

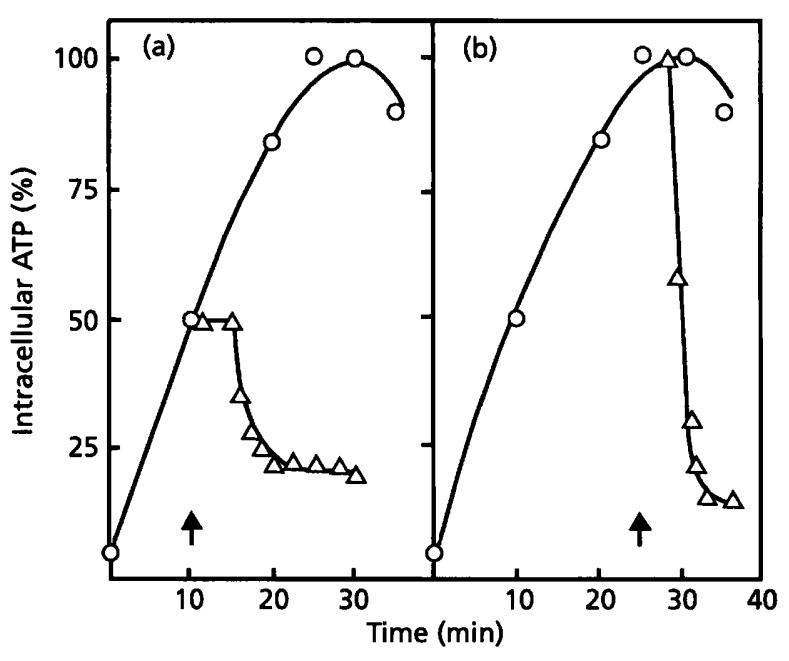

Fig. 2

Fig. 1. Membrane potential changes induced by carnocin UI49. The membrane potential was measured as described in Methods. Experiments were performed at $30^{\circ} \mathrm{C}$ in $1.0 \mathrm{ml} 50 \mathrm{mM}$ phosphate buffer $\mathrm{pH} 6.0$. At the points indicated by the arrows the following additions were made: (1) $0.1 \mathrm{ml}$ cell suspension; (2) glucose, final concentration $0.18 \%$ (w/v); (3) nigericin, final concentration $0.36 \mu \mathrm{M}$; and (4) carnocin UI49, final concentration $8.5 \mathrm{mg} \mathrm{I}^{-1}(\longrightarrow$ ) or valinomycin, final concentration $0.72 \mu \mathrm{M}(---)$.

Fig. 2. Effect of carnocin UI49 on intracellular ATP levels in L. lactis NZ9700. The ATP content was measured as described in Methods. The assay was performed in $50 \mathrm{mM}$ phosphate buffer $\mathrm{pH}$ 6.0. At time zero the cells were energized with glucose (final concentration $0.5 \%, w / v)$. Values are presented as a percentage of the maximum intracellular ATP concentration $(100 \%$ is $10 \mathrm{mM})$. The change in the ATP level in the cells was monitored with $(\triangle)$ or without $(O)$ addition of carnocin UI49 (final concentration $6.3 \mathrm{mg} \mathrm{l}^{-1}$ ) after $10 \mathrm{~min}$ (a) or $25 \mathrm{~min}$ (b) of incubation at $30^{\circ} \mathrm{C}$ (as indicated by arrows).

NCDO497 was grown overnight in GM17 at $30^{\circ} \mathrm{C}$ and added to $50 \mathrm{mM}$ sodium phosphate buffer, $\mathrm{pH} 6.5$, with and without carnocin UI49 (1000 bacteriocin units $\mathrm{ml}^{-1}$ ). Viable cells were determined after incubation of the samples at $4{ }^{\circ} \mathrm{C}, 15^{\circ} \mathrm{C}, 22^{\circ} \mathrm{C}, 30^{\circ} \mathrm{C}$ and $34^{\circ} \mathrm{C}$. The results suggested that maximum bactericidal activity of carnocin UI49 occurred at $34^{\circ} \mathrm{C}$ with a decrease in activity down to $15^{\circ} \mathrm{C}$ where it was completely abolished (Stoffels et al., $1992 \mathrm{~b})$.

In this study, L. lactis subsp. lactis NZ9700 was cultured twice at $12{ }^{\circ} \mathrm{C}$ and $30{ }^{\circ} \mathrm{C}$ before assaying its sensitivity to carnocin UI49 at high and low temperatures. The diameters of the inhibition zones when assayed at $12{ }^{\circ} \mathrm{C}$ and $30^{\circ} \mathrm{C}$ were 33 and $27 \mathrm{~mm}$, respectively. These results clearly demonstrate that carnocin UI49 is indeed also active at low temperatures. Growth of the indicator strain at low temperatures is apparently accompanied by the adaption of the cytoplasmic membrane such that membrane fluidity is maintained allowing insertion of and channel formation by carnocin UI49 resulting in growth inhibition at low temperature.

\section{Effect of carnocin UI49 on the permeability of the cytoplasmic membrane of non-nisin-producing $L$. lactis R520}

Thus far, all experiments with carnocin UI49 were performed with nisin-producing $L$. lactis indicator strains. These strains have been the indicator strains of choice,
Table 2. Strains of $L$. lactis subsp. lactis tested for sensitivity to carnocin UI49 and immunity to nisin Z

Values represent the means of five measurements.

\begin{tabular}{|lcc|}
\hline Indicator strain & $\begin{array}{c}\text { Immunity to } \\
\text { nisin Z* } \\
(\%)\end{array}$ & $\begin{array}{c}\text { Sensitivity to } \\
\text { carnocin UI49† } \\
(\%)\end{array}$ \\
\hline L. lactis subsp. lactis & & \\
NZ9700 & 100 & 100 \\
R520 & 6 & 6 \\
MG1614(pNZ9111) & 3 & 1 \\
NZ9800 & 10 & 11 \\
MG1614 & 0.09 & 1.5 \\
\hline
\end{tabular}

* $100 \%$ immunity of L. lactis subsp. lactis NZ9700 to nisin Z: $3.3 \mathrm{mg} \mathrm{l}^{-1}$.

$+100 \%$ sensitivity of $L$. lactis subsp. lactis NZ9700 to carnocin U149: $0.09 \mathrm{mg} \mathrm{l}^{-1}$.

because they are at least 10 -fold more sensitive to carnocin UI49 than the non-nisin-producing LAB (Stoffels $e t$ al., 1993). Dissipation of the membrane potential was not observed for the less sensitive cells of $L$. lactis subsp. lactis R520 (Table 2) after addition of carnocin UI49 to a final concentration of $34 \mathrm{mg} \mathrm{l}^{-1}$ (data not shown). Due to the 


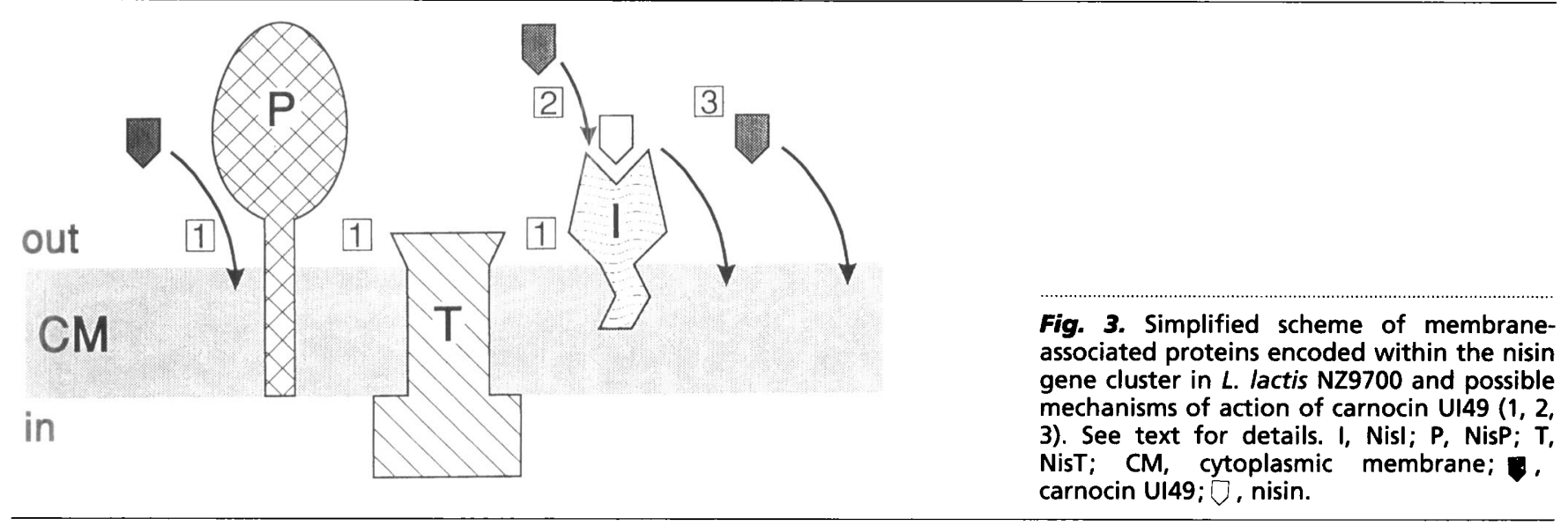

high salt concentration of the bacteriocin samples $(0 \cdot 2 \mathrm{M}$ $\mathrm{NaCl}$ ), it was not possible to add more carnocin UI49 to the cell solution without changing the experimental conditions. Unfortunately, the sample could not be desalted without a reduction in bacteriocin activity of at least $90 \%$. This could be due to a decrease in the solubility of carnocin UI49. The loss in activity may also be due to the aggregation of carnocin UI49 molecules as was observed for nisin (Liu \& Hansen, 1990).

\section{Possible role of membrane-associated proteins in the sensitivity of nisin-producing lactococcal cells to carnocin U149}

The reason for the high sensitivity of the nisin-producing L. lactis subsp. lactis strains to carnocin UI49 was studied further. The results of this study indicate that carnocin UI49 acts at the cytoplasmic membrane and the high sensitivity of the nisin-producing cells may be due to proteins encoded within the nisin gene cluster. Three membrane-associated proteins have been described that are involved in nisin biosynthesis and immunity to nisin. NisI is a lipid-modified extracellular membrane-anchored protein of $32 \mathrm{kDa}$ that is involved in the immunity of the nisin producer strain to nisin (Kuipers et al., 1993). NisP is a subtilisin-like serine protease of $54 \mathrm{kDa}$ that cleaves off the leader sequence from fully matured precursor nisin. The protease is attached to the cellular membrane via a C-terminal anchor sequence (Van der Meer et al., 1993). NisT is a $69 \mathrm{kDa}$ ATP-dependent membrane translocator which is involved in nisin transport (Blight \& Holland, 1990; Engelke et al., 1992). These proteins are encoded on a lactococcal gene cluster involved in nisin biosynthesis, immunity and genetic mobility (Klaenhammer, 1993). This gene cluster is located on the conjugative transposon Tn5276. This element also encodes the genes involved in sucrose utilization (Rauch \& De Vos, 1992). The fermentation of sucrose depends initially upon the translocation and phosphorylation of the sugar mediated by the membrane-associated phosphoenolpyruvate-dependent sucrose: phosphotransferase system (sucrose-PTS) (Thompson et al., 1991). The expression of the suc genes may be under glucose (catabolite) repression control in a manner similar to the lac genes (Poolman, 1993). Therefore, in order to repress the expression of the sucrose-PTS, all $L$. lactis subsp. lactis strains were grown in BHI supplemented with glucose.

Fig. 3 shows how NisI, NisP and NisT may be inserted into the lactococcal cell membrane. Furthermore, the model shows possible ways in which these proteins may be involved in the bactericidal activity of carnocin UI49. The first mechanism (1) shows how one of the proteins could serve as a receptor for carnocin UI49 and facilitate the binding and/or subsequent insertion of carnocin UI49 into the membrane. This may increase the efficiency of bactericidal activity. The second mechanism shows another possibility for NisI involvement (2). NisI may neutralize the bactericidal activity of nisin by interception of the nisin molecules. This would explain the limited immunity of the wild-type nisin-producing strains to a high concentration of nisin. Carnocin UI49 could compete with the nisin molecules for the same site on NisI, thereby releasing nisin. Nisin kills cells more efficiently than carnocin UI49; the minimum inhibitory concentration of nisin against $L$. lactis subsp. lactis MG1614 is $0.003 \mathrm{mg} \mathrm{l}^{-1}$ while the minimum inhibitory concentration of carnocin UI49 against $L$. lactis subsp. lactis NZ9700 is $0.09 \mathrm{mg} \mathrm{l}^{-1}$ (Table 2). Therefore, killing of the lactococcal cells by this mechanism would be mainly the result of pore formation by the nisin molecules that have been released from NisI. The third mechanism (3) does not involve proteins, but is based upon differences in the phospholipid composition and/or other characteristics of the cytoplasmic membrane as compared to that of non-nisin-producing $L$. lactis subsp. lactis strains.

In order to elucidate which of the above is the most plausible mode of action of carnocin UI49, the latter was tested against various transformed $L$. lactis subsp. lactis strains. The L. lactis subsp. lactis MG1614 strain used has been transformed with the plasmid pNZ9111 which contains all the genes involved in nisin biosynthesis and immunity with the exception of nisP (Van der Meer et al., 1993). Consequently strain L. lactis subsp. lactis MG1614(pNZ9111) produces fully matured inactive extracellular precursor nisin. The other mutant used, $L$. lactis subsp. lactis NZ9800, contains the complete Tn5276, but is deficient in nisin A production due to gene replacement of $n i s A$ by a truncated nis $A$ gene (Kuipers $e t$ al., 1993). The third mutant used, L. lactis subsp. lactis 
Table 3. Strains of Lb. sake and S. epidermidis tested for sensitivity to carnocin UI49

Values represent the means of two measurements.

\begin{tabular}{|lc|}
\hline Indicator strain & $\begin{array}{c}\text { Sensitivity to carnocin } \\
\text { UI49 }\left(\mathrm{mg} \mathrm{l}^{-1}\right)\end{array}$ \\
\hline Lb. sake & $0 \cdot 7$ \\
LMG2340 & $1 \cdot 4$ \\
LMG2346 & \\
S. epidermidis $_{5}$ & $>50$ \\
5 & $>50$ \\
5Pep5 & $>50$ \\
\hline
\end{tabular}

R520 has a mutated $\operatorname{Tn} 5276$, which has not been further specified (Kuipers et al., 1993).

The sensitivities of the three lactococcus mutants to carnocin UI49 were reduced as compared to the wild-type nisin-producing strain (Table 2). However, the sensitivities of L. lactis subsp. lactis R520 and L. lactis subsp. lactis NZ9800 were still 4-fold and 7-fold higher, respectively, than the sensitivity of the non-nisin-producing plasmid-free wild-type strain $L$. lactis subsp. lactis MG1614. These results indicate that the sensitivity of the wild-type nisin-producing strains to carnocin UI49 cannot be explained by mechanism 2 (Fig. 3), since these mutants do not produce active nisin but are still more sensitive to carnocin UI49 than is L. lactis subsp. lactis MG1614.

The ability of some $L$. lactis subsp. lactis strains to produce nisin is tightly coupled to their immunity to nisin; full immunity is observed only when matured nisin is produced (Kuipers et al., 1993). The expression of all genes involved in the biosynthesis of and immunity to nisin may be linked. The immunity of the L. lactis subsp. lactis strains was measured as an indication of the expression of the nisin gene cluster and thus the presence of NisT, NisP and NisI. The results are shown in Table 2. Indeed, the decrease in immunity of $L$. lactis subsp. lactis R520, L. lactis subsp. lactis NZ9800 and L. lactis subsp. lactis MG1614(pNZ9111) to nisin corresponded to the decrease in sensitivity to carnocin UI49. This suggests that carnocin UI49 may act according to mechanism 1 (Fig. 3), i.e. receptor-mediated induction of carnocin UI49 activity whereby NisI, Nis'T and/or NisP function as a receptor.

L. lactis subsp. lactis MG1614(pNZ9111) showed the highest decrease in sensitivity to carnocin UI49 and immunity to nisin relative to the wild-type nisin-producing strain. Its immunity to nisin is still 30-fold higher than that of L. lactis subsp. lactis MG1614, the wild-type non-nisin-producing strain. This suggests that $L$. lactis subsp. lactis MG1614(pNZ9111) still harbours the immunity system against nisin. L. lactis subsp. lactis MG1614 shows similar sensitivity to carnocin UI49 as L. lactis subsp. lactis MG1614(pNZ9111), which suggests that the latter has lost the factor that induces the activity of carnocin UI49. In contrast to the other transformed $L$. lactis subsp. lactis strains or the wild-type nisin-producing strain, this strain does not contain the gene encoding NisP. Therefore, if carnocin UI49 acts according to mechanism 1 (Fig. 3), NisP is the most likely candidate to function as a receptor. The last $30 \mathrm{C}$-terminal amino acid residues of NisP show homology with a number of membrane-anchoring attachment sites of proteins of Gram-positive bacteria. These membrane-spanning sequences contain a hydrophobic $\alpha$-helix with the hydrophilic residues located at one site (Vos et al., 1989; Van der Meer et al., 1993). If the NisP attachment site is similar, one could envisage the involvement of this site in the facilitated insertion of carnocin UI49 into the membrane.

\section{Activity of carnocin UI49 against other lantibiotic- producing strains}

Table 3 shows the bactericidal activity of carnocin UI49 against two other lantibiotic-producing strains, $L b$. sake and S. epidermidis. Lb. sake LMG2346 ( $\left.\mathrm{Lac}^{-} \mathrm{Imm}^{-}\right)$ showed similar sensitivity towards carnocin UI49 as the wild-type producer strain $L b$. sake LMG2340 $\left(\mathrm{Lac}^{+}\right.$ $\mathrm{Imm}^{+}$). Lb. sake LMG2346 has been cured for the plasmid containing the genes that are involved in lactocin $\mathrm{S}$ immunity and production. Since this drastic mutation does not affect the sensitivity towards carnocin UI49 our results suggest that lactocin $\mathrm{S}$ production and/or immunity is not coupled to carnocin UI49 sensitivity. Finally, S. epidermidis, the Pep5 producer strain and its derivatives were not sensitive to carnocin UI49.

\section{Conclusion}

This paper presents results on the mode of action of carnocin UI49. Carnocin UI49 increased the permeability of the cytoplasmic membrane of $L$. lactis subsp. lactis NZ9700 in a manner similar to other bacteriocins. The high sensitivity of the nisin-producing $L$. lactis subsp. lactis to carnocin UI49 relative to other $L$. lactis subsp. lactis strains was further investigated. One of the membrane-associated proteins involved in the production of nisin or the immunity to nisin seemed to be involved in the sensitivity of these cells to carnocin UI49. Furthermore, the results suggested that NisP may act as a receptor for carnocin UI49, thereby increasing carnocin UI49 activity. It is not yet known whether the phospholipid composition of the nisin-producing $L$. lactis subsp. lactis strains differs from that of the non-nisin producing L. lactis subsp. lactis strains. Cloning and expression of each of the genes involved in nisin production and immunity to nisin may show whether NisP is the only protein which induces carnocin UI49 activity and possibly acts as its receptor.

C. piscicola UI49, the carnocin UI49 producer strain, is as sensitive to nisin as other carnobacteria (Stoffels $e t$ al., 1993). Furthermore, the lantibiotic-producing and nonproducing mutants of $S$. epidermidis and $L b$. sake did not show a difference in sensitivity to carnocin UI49. The proposed receptor-mediated action of carnocin UI49 at the cytoplasmic membrane therefore seems to be specific 
for carnocin UI49 and the nisin-producing L. lactis subsp. lactis strains.

\section{ACKNOWLEDGEMENTS}

We thank Jeroen Hugenholtz and Oscar Kuipers, Department of Microbiology, Netherlands Institute for Dairy Research (NIZO), Ede, The Netherlands, Ingolf Nes, Laboratory of Microbial Gene Technology, Ås, Norway, and Hans-Georg Sahl, Institute for Medical Microbiology and Immunology, University of Bonn, Germany for providing us with the indicator strains. This work was supported by grant P89047 from the Nordic Fund for Technology and Industrial Development and grant $0134 \mathrm{~S}$ from the National Research Council in Iceland.

\section{REFERENCES}

Abee, T., Gao, F. H. \& Konings, W. N. (1991). The mechanism of action of the lantibiotic nisin in artificial membranes. In Nisin and Novel Lantibiotics, pp. 373-385. Edited by G. Jung \& H.-G. Sahl. Leiden: Escom.

Andersson, R. (1986). Inhibition of Staphylococcus aureus and spheroplasts of Lactobacillus plantarum. Int J Food Microbiol 3, 149-160.

Anonymous (1989). Countries where specific approval exists for the use of nisin. Technical Information Leaflet no. 4/89/11. Aplin and Barrett Ltd.

Benz, R., Jung, G. \& Sahl, H.-G. (1991). Mechanism of channel formation by lantibiotics in black lipid membranes. In Nisin and Novel Lantibiotics, pp. 422-433. Edited by G. Jung \& H.-G. Sahl. Leiden: Escom.

Blight, M. A. \& Holland, I. B. (1990). Structure and function of haemolysin B, P-glycoprotein and other members of a novel family of translocators. Mol Microbiol 4, 873-880.

De Vos, W. M., Mulders, J. W. M., Siezen, R. J., Hugenholtz, J. \& Kuipers, O. P. (1993). Properties of nisin $\mathrm{Z}$ and distribution of its gene, nisZ, in Lactococcus lactis. Appl Environ Microbiol 59, 213-218. Delves-Broughton, J. (1990). Nisin and its uses as a food preservative. Food Technol 44, 100-117.

Driessen, A. J. M., Kodde, J., de Jong, S. \& Konings, W. N. (1987). Neutral amino acid transport by membrane vesicles of Streptococcus cremoris is subjected to regulation of the internal pH.J Bacteriol 169, 2748-2754.

Engelke, G., Gutowski-Eckel, Z., Hammelmann, M. \& Entian, K.-D. (1992). Biosynthesis of the lantibiotic nisin: genomic organization and membrane localization of the NisB protein. Appl Environ Microbiol 58, 3730-3743.

Gao, F. H., Abee, T. \& Konings, W. N. (1991). Mechanism of action of the peptide antibiotic nisin in liposomes and cytochrome $c$ oxidase-containing proteoliposomes. Appl Environ Microbiol 57, 2164- 2170.

Garcera, M. J. G., Elferink, M. G. L., Driessen, A. J. M. \& Konings, W. N. (1993). In vitro pore-forming activity of the lantibiotic nisin. Role of protonmotive force and lipid composition. Eur $J$ Biochem 216, 417-422.

Gasson, M. J. (1983). Plasmid complements of Streptococcus lactis NCDO 712 and other lactic streptococci after protoplast-induced curing. J Bacteriol 154, 1-9.

Guihard, G., Bénedetti, H., Besnard, M. \& Letellier, L. (1993). Phosphate efflux through the channels formed by colicins and phage T5 in Escherichia coli cells is responsible for the fall in cytoplasmic ATP. J Biol Chem 268, 17775-17780.
Hoover, D. \& Steenson, L. (1993). Bacteriocins of Lactic Acid Bacteria. New York: Academic Press.

Jung, G. (1991). Lantibiotics: a survey. In Nisin and Novel Lantibiotics, pp. 1-34. Edited by G. Jung \& H.-G. Sahl. Leiden: Escom.

Klaenhammer, T. R. (1993). Genetics of bacteriocins produced by lactic acid bacteria. FEMS Microbiol Rev 12, 39-86.

Kuipers, O. P., Beerthuyzen, M. M., Siezen, R. J. \& De Vos, W. M. (1993). Characterization of the nisin gene cluster nis ABTCIPR of Lactococcus lactis; requirement of expression of the nis A and nis genes for development of immunity. Eur J Biochem 216, 281-291.

Liu, W. \& Hansen, J. N. (1990). Some chemical and physical properties of nisin. Appl Environ Microbiol 56, 2551-2558.

Mørtvedt, C., Nissen-Meyer, J., Sletten, K. \& Nes, I. F. (1991). Purification and amino acid sequence of lactocin $\mathrm{S}$, a bacteriocin produced by Lactobacillus sake L45. Appl Environ Microbiol 57, 1829-1834.

Ojcius, D. M. \& Young, D.-E. (1991). Cytolytic pore-forming proteins and peptides: is there a common structural motive? Trends Biocbem Sci 16, 225-229.

Piard, J.-C., Muriana, P. M., Desmazeaud, M. J. \& Klaenhammer, T. R. (1992). Purification and partial characterization of lacticin 481 , a lantionine-containing bacteriocin produced by Lactococcus lactis subsp. lactis CNRZ 481. Appl Environ Microbiol 58, 279-284.

Poolman, B. (1993). Energy transduction in lactic acid bacteria. FEMS Microbiol Rev 12, 125-148.

Rauch, P. J. G. \& De Vos, W. M. (1992). Characterization of the novel nisin-sucrose conjugative transposon Tn5276 and its insertion in Lactococcus lactis. J Bacteriol 174, 1280-1287.

Rauch, P. J. G., Beerthuyzen, M. M. \& De Vos, W. M. (1991). Molecular analysis and evolution of conjugative transposons encoding nisin production and sucrose fermentation in Lactococcus lactis. In Nisin and Novel Lantibiotics, pp. 243-249. Edited by G. Jung \& H.-G. Sahl. Leiden: Escom.

Ray, B. (1992). Bacteriocins of starter culture bacteria as food preservatives: an overview. In Food Preservatives of Microbial Origin, pp. 178-205. Edited by B. Ray \& M. Daeschel. Florida: CRC Press.

Reis, M. \& Sahl, H.-G. (1991). Genetic analysis of the producer selfprotection mechanism ("immunity") against Pep-5. In Nisin and Novel Lantibiotics, pp. 320-331. Edited by G. Jung \& H.-G. Sahl. Leiden: Escom.

Ruhr, E. \& Sahl, H.-G. (1985). Mode of action of the peptide antibiotic nisin and the influence on the membrane potential of whole cells and on cytoplasmic and artificial membrane vesicles. Antimicrab Agents Chemother 27, 841-845.

Sahl, H.-G., Kordel, M. \& Benz, R. (1987). Voltage dependent depolarization of bacterial membranes and artificial lipid bilayers by the peptide antibiotic nisin. Arch Microbiol 149, 120-124.

Shinbo, T., Kama, N., Karihara, K. \& Kobataka, Y. (1978). PVCbased electrode sensitive to $\mathrm{TPP}^{+}$as a device to monitor the membrane potential in biological systems. Arch Biochem Biophys 187, 414-422.

Stoffels, G., Nissen-Meyer, J., Guðmundsdóttir, Á., Sletten, K., Holo, H. \& Nes, I. F. (1992a). Purification and characterization of a new bacteriocin isolated from a Carnobacterium sp. Appl Environ Microbiol 58, 1417-1422.

Stoffels, G., Nes, I. F. \& Guðmundsdóttir, Á. (1992b). Isolation and properties of a bacteriocin-producing Carnobacterium piscicola isolated from fish. J Appl Bacteriol 73, 309-316.

Stoffels, G., Sahl, H.-G. \& Guð̀mundsdóttir, A. (1993). Carnocin UI49, a potential biopreservative produced by Carnobacterium 
piscicola. Large scale purification and activity against various Grampositive bacteria including Listeria sp. Int $J$ Food Microbiol 20, 199-210.

Thompson, J., Sackett, D. L. \& Donkersloot, J. A. (1991). Purification and properties of fructokinase I from Lactococcus lactis. Localization of scrK on the sucrose-nisin transposon Tn5306. J Biol Chem 266, 22626-22633.

Van der Meer, J. R., Polman, J., Beerthuyzen, M. M., Siezen, R. J., Kuipers, O. P. \& De Vos, W. M. (1993). Characterization of the Lactococcus lactis nisin A operon genes nis $\mathrm{P}$, encoding a subtilin-like serine protease involved in precursor processing, and $n i s \mathrm{R}$, encoding a regulatory protein involved in nisin biosynthesis. $J$ Bacteriol 175, 2578-2588.

Venema, K., Abee, T., Haandriksman, A. J., Leenhouts, K. J., Kok,
J., Konings, W. N. \& Venema, G. (1993). Mode of action of lactococcin B, a thiol-activated bacteriocin from Lactococcus lactis. Appl Environ Microbiol 59, 1041-1048.

Vos, P., Simons, G., Siezen, R. J. \& De Vos, W. M. (1989). Primary structure and organization of the gene for a procaryotic, cell envelope-located serine protease. J Biol Chem 264, 13579-13585.

Zaman, Z. \& Verwilghen, R. L. (1979). Quantitation of proteins solubilized in sodium dodecyl sulfate-mercaptoethanol-Tris electrophoresis buffer. Anal Biochem 100, 64-69.

Received 15 November 1993; revised 4 January 1994; accepted 10 January 1994 